\title{
The Effects of Cleaning Solutions on Bacteria Concentra- tion on Hospital Wheelchairs
}

\author{
Erin Knapp ${ }^{1}$ \\ ${ }^{1}$ Mentor High School, Mentor, Ohio, USA
}

\begin{abstract}
$\underline{\text { ABSTRACT }}$
To what extent does the concentration of bacteria located on hospital wheelchairs vary based on the cleaning solution used between patient transport? The purpose of this study was to investigate the cause of HAIs [Hospital Acquired Infections] in hospital environments and determine the best cleaning method of wheelchairs in hospitals. The method was to swab the seats and handles of wheelchairs before and after cleaning them while wearing gloves in comparison to no gloves with varying strengths of cleaning solutions, plating the swab, and allowing the bacteria to be incubated and grow in an incubator. Then, every day for 10 days, plates were measured on a piece of graph paper to record growth. A one-way ANOVA was calculated for the effectiveness of the various cleaners and a p-value of 0.6597 was achieved and a t-test was performed to compare the values of gloves versus no gloves and a p-value of 0.2216 was calculated. The p-values were not significant at 0.05 . Overall, wearing gloves while cleaning showed to have a slight positive impact on the overall cleanliness but was not statistically significant, and the highest strength wipes inhibited bacterial growth most often. Hospital protocol for the cleansing of wheelchairs between patient use should be updated to the use of gloves and high strength wipes to stop the spread of bacterial infections to patients.
\end{abstract}

\section{Introduction}

Hospital acquired infections, also known as HAIs or nosocomial infections, have become a prevalent issue in modernday hospitals. Defined as an infection that appears 48 hours after hospital admission, and therefore is acquired within the admission time period, HAIs are often transmitted due to a fault in cleaning protocol (Revelas, 2012). Some of the most common nosocomial infections include pneumonia, urinary tract infections, and sepsis ("13 most common", 2014). Although present throughout history, nosocomial infections, infections originating in a hospital, have recently been on the rise in the recent body of knowledge. Specifically, in recent medical reports, European hospitals have disclosed that roughly five million cases of hospital acquired infections have occurred within some of their acute care hospitals. More frighteningly, these nosocomial infections resulted in a mortality rate of over 50,000 patient deaths (Arefian, et al., 2019). In more recent times, nosocomial infections are still prevalent. In 2015, there were roughly 687,000 HAIs in U.S. acute care hospitals. Of those, about 72,000 hospital patients with hospital acquired infections died during their hospitalizations (CDC, 2020). To fight hospital acquired infections, many hospitals began using Chlorine-based cleaning solutions, before turning to Polyphenolic cleaning solutions after WWII. Polyphenols are known as various alcohols and are commonly plant based. A few common polyphenols are triclosan and chloroxylenol, biocides used both commercially and by hospitals ("Phenolics", 2020; Tessarolo et al., 2007). Later in the 1980s, hospitals opted for chemically-treated, cotton mops. These mops used both a dust removing chemical alongside antimicrobial agents (Vesley, Klapes, Benzow, \& Le, 1987). However, recent hospitals have been turning towards bleach and other chemically based cleaners. Now, most hospitals have implemented a stronger cleaning protocol. Multiple hospitals have opted for anti-bacterial cleansing wipes to ensure a decrease in bacteria in patient high-contact areas such as patient rooms, waiting areas, and treatment rooms (Arefian, et al., 2019). While this cleaning method has reduced the number of nosocomial infections, using the same wipe on multiple surfaces may not only be seen as 
ineffective but also may aid in the spread of bacteria from surface to surface (Walsh, 2008). This practice, along with other unsafe cleaning protocols, has contributed to the increase in hospital acquired infections.

In recent times, the spread of the novel "coronavirus", also known scientifically as SARS-COVID-2 or COVID-19, has been worrying healthcare providers and patients alike. COVID-19 has raised concerns due to its rapid spread through contact with surfaces alongside person to person contact (Rothan \& Byrareddy, 2020). The spread of this virus through contact with surfaces has raised concerns with the cleanliness of surfaces within the hospital to ensure the safety of individuals within the hospital environment. It must be noted that COVID-19 is a viral infection, and this study focuses more on the concentration of bacterial infections and the presence of bacteria on low contact surfaces, wheelchairs in particular.

The current climate surrounding hospital cleaning processes focuses highly on sterilizing high-contact materials, such as catheters, bed railings, and light switches found in patient's rooms (Han et al., 2015). Most journals in the field of my research, cleanliness of hospitals and the contribution of surface bacteria to the spread of diseases, discuss the prevalence of hospital acquired infections in terms of high-contact surfaces as previously stated, without many considerations to areas with low-contact such as wheelchairs, nurse's stations, and room decor. In defining terms, low contact areas are surfaces that are not as often in skin to skin contact with patients. High contact areas, in contrast, are defined as areas that are regularly in skin to skin contact with patients. Similarly, many studies focused on limiting the rate in which nosocomial infections spread, rather than addressing the specific cleaning protocol. This prevents the spread of bacteria not only to patients but also to others present in the hospital setting such as families and health care facility workers. To clarify, hospital acquired infections only specifically refer to hospital patients who become infected while being treated in the hospital. These studies do not account for the infection of hospital staff or families of patients.

In terms of COVID-19, researchers are tirelessly working to investigate disinfecting techniques. As stated by Hadis Fathizadeh et al. in "Protection and Disinfection Policies Against SARS-CoV-2 (COVID-19)," It has been proven that disinfectants with $62-71 \%$ ethanol or $0.1 \%$ sodium hypochlorite are able to reduce the contamination of coronavirus on surfaces within one minute of exposure (Fathizadeh et al., 2020). A few examples of products that contain these disinfectants are Purell hand sanitizer and Lysol. However, more research must be done to research the persistence of COVID-19 on various surface times. This study does not mention the cleaning methods in terms of high and low contact areas, but surfaces as a whole.

There is also a lack of research conducted on the role of low-contact objects in the spread of hospital acquired infections. While some researchers suggest there may be a connection between two factors, they fail to elaborate on the role of these objects. As suggested by researcher G. Velvizhi in "Wristwatches as the Potential Sources of HospitalAcquired Infections," many low-contact objects, including the wristwatches of staff members, are not cleaned as often as high-contact objects, leading to an increased spread of bacteria (Velvizhi et al. 2012). This connects with the idea of wheelchair cleaning methods, as hospital transport staff are often handling wheelchairs, and fail to wipe the handles of the wheelchair between patients along with disregarding participating in active handwashing practices, unlike most hospital staff. Even if other areas of wheelchairs are cleaned regularly, for example, the seat and armrests, handles, and other individual parts of wheelchairs are not as regularly cleaned. These parts of the wheelchair may harbor more bacteria that could contribute to the spread of disease and illness among individuals visiting the hospital. Wheelchairs are often used throughout the hospital, making them even more susceptible fomites, inanimate objects that, when contaminated with or exposed to infectious agents, can transfer disease to a new host. to infections. Also, the cleanliness of the hands of workers could impact bacteria spread and infections. As stated by William Rutala, the contamination of the healthcare worker's hands via patients or indirect contact with contaminated surfaces could contribute to the spread of bacteria (Rutala \& Weber, 2014). This article calls for further research into the impact of hand contamination. This is where more research is able to fill a gap, as it focuses on the cleaning method of wheelchairs, lowcontact objects. As researched multiple times, hand washing and sterile gloves make all contact more sanitary than the alternative. As stated by nursing researcher A. Dziewa in "Nurse care quality and hospital-acquired infections: adhering to aseptic techniques," medical staff who lacked proper hygiene protocol such as the use of glove and 
improper handwashing techniques contributed to the spread of HAIs (Dziewa, Ksykiewicz-Dorota, Kos \& Drop, 2015). Therefore, it is important to investigate the implications of having clean hands when washing wheelchairs. Other studies have shown gloves to be a positive preventative measure in inhibiting bacterial spread, however, these studies only applied towards frontline healthcare workers, rather than other hospital staff (Moore, 2018). The cleaning methods will also be tested with gloves on, as well as without gloves to study the impact of contaminated hands on the bacteria count. Alongside the beneficial aspect of wearing gloves on bacterial concentrations, wearing gloves while cleaning wheelchairs provides many other benefits so staff members. Particularly, the use of gloves while cleaning will limit the contact between the skin of the workers and bacteria that may be present on the surface itself. These protective, sterile gloves stop not only the spread of bacteria from the staff member to the surface, but also stops harmful bacteria that may be present from coming in contact with the hospital staff member (Lopez-Alcalde et al., 2008).

The purpose of this study is to investigate the cause of HAIs in hospital environments and determine the best cleaning method of wheelchairs in hospitals. By studying the effectiveness of methods of cleaning, wheelchairs being used in hospitals will be unlikely to increase the rate of nosocomial infections alongside establishing the best cleaning practice of transportation devices (Dancer, White \& Robinson, 2008). By establishing the most effective cleaning processes, both patients and hospital staff's protection against bacterial infections is greatly increased. In the same vein, by evaluating different cleaning methods, a new protocol is able to be put into place within numerous hospitals to ensure the safety of hospital patients, patient's families, and hospital staff members. Finally, this led me to the research question: to what extent does the concentration of bacteria located on hospital wheelchairs vary based on the cleaning solution used between patient transport?

\section{Methods}

The study employed a quantitative, experiment/quasi-experiment method. The purpose of this experiment was to investigate the cause of HAIs [Hospital Acquired Infections] in hospital environments by monitoring bacterial growth before and after cleaning protocol has occurred. Bacterial growth occurred in Petri dishes to analyze the growth rate over time. While the use of an electron microscope and other higher-tech lab equipment would have enhanced the results of the experiment, the use of Petri dishes allowed for a simple comparison tool of bacterial growth between the variables being tested. Petri dishes were also the most effective option for student access, as they are common lab equipment not only used by students, but also scientists in the lab. Seats were swabbed in order to show the effects of bacteria that may be in contact with patients, while handles were swabbed to show the impact on staff members who are transporting patients. If a relationship between the cleaning protocol used and bacterial growth is present, this new protocol can be implemented into regular hospital protocol for the cleaning of wheelchairs between patient use.

I suspect that there will be overall a lesser amount of bacterial growth on the plate with wipes containing a high concentration of bleach and quaternary ammonium such as the Sani-Cloth and Sani-Cloth Bleach wipes (Russotto, Cortegiani, Fasciana, et. al., 2017). In the same vein, wheelchairs that are cleaned while wearing sterile gloves will also house a smaller concentration of bacteria in comparison. However, as advised by A. Bello, M. Quinn, M. Perry $\&$ D. Milton in the study "Characterization of occupational exposures to cleaning products used for common cleaning tasks--a pilot study of hospital cleaners," caution must be exercised when cleaning areas without the use of gloves, as bleach and other concentrated chemicals may lead to skin irritation and chemical burns if exposed for long periods of time (Bello, Quinn, Perry, Milton, 2009).

\section{Experimental Manipulations}

In order to begin the experiment, variables were defined. The cleaning supplies used was the independent variable, while the bacterial growth was the dependent variable. The experiment was self-delivered at a local medical center. The medical center is a local suburban community hospital that is private and not-for-profit. It is a four-story 
hospital that can house about 119 patients in their private patient rooms alongside 22 emergency trauma treatment rooms and various other medical subspecialty wards. Ten Petri dishes were used and divided in half to produce 20 samples in totality. These dishes were further monitored over a span of eight days.

\section{Measures and Covariates}

A hypothesis was formulated that bleach-based cleaners, specifically the Sani-Cloth Bleach wipes, would be the most effective in the inhibition of bacteria growth. In the same vein, a hypothesis was formed that wearing gloves during the cleaning procedure would have a positive impact on the cleanliness of the wheelchairs. The primary data collected was the overall effectiveness of various cleaning products on the inhibition of bacterial growth. However, secondary data was also collected. The secondary data collected was the impact of wearing gloves during cleaning protocol has on the bacteria concentration after cleaning.

\section{Procedure}

Data was collected on January 19th, 2020 in the basement of the hospital, in which wheelchairs are normally stored between use. There was a 5 minute period between the use of the wheelchair by the patient and data collection. Pre-made nutrient agar Petri dishes were purchased from LabExpress based in Michigan and contained 0.3\% Beef Extract, $0.5 \%$ Peptone, and $1.5 \%$ Agar to provide a food source for the growing bacterial culture and were $150 \times 15 \mathrm{~mm}$ each. Agar Petri dishes infused with beef broth provide nutrients that aid in bacterial growth and reproduction. Sterile Cotton Tip Applicators were purchased from Dynarex through Amazon. It was crucial that sterile supplies were used in order to ensure no outside bacteria contaminated the dishes while swabbing occured. Three different cleaning supplies were tested: Virex II 256 spray (Spray and rag), Sani-Cloth (low-strength wipe), and Sani-Cloth Bleach (highstrength wipe). The three cleaning products were used due to their current regular usage throughout the hospital. Virex II 256 is a one-step disinfectant cleaner and deodorant. Its main active ingredients are Didecyl dimethyl ammonium chloride n-Alkyl and dimethyl benzyl ammonium chloride, both of which are common antiseptic cleaners used by hospitals. Quaternary ammonium compounds and bleach based compounds include cleaning products such as Clorox wipes, Lysol sprays, and Mr. Clean anti-bacterial sprays. Didecyl dimethyl ammonium chloride n-Alkyl and other quaternary ammonium compounds, as stated by Christopher Ioannou, Geoff Hanlon, and Stephen Denyer of the School of Pharmacy and Biomolecular Sciences at the University of Brighton, cause damage to the cellular membrane of bacteria cells (Ioannou, Hanlon \& Denyer, 2006). By damaging the cell membrane, bacterial cells fail to reproduce and die, therefore preventing further spread and infection. Sani-Cloth is a germicidal disposable wipe and its main active ingredients are the same as Virex II 256, with the only difference being that they are found in different concentrations (See Appendix I). Finally, Sani-Cloth Bleach is also a germicidal disposable wipe and its main active ingredient is sodium hypochlorite, commonly known as bleach. As stated by Heidi Ledford in Nature magazine, bleach denatures crucial proteins made by bacteria cells, which causes bacterial death and inhibits the reproduction of bacteria (Ledford, 2008).

The same wheelchair was used for each test to ensure there would be no variability of cultures from different chairs. Each cleaning product was tested twice: once with the use of gloves and once without the use of gloves. Both the seat and handles were tested with each cleaning product. The cleaning process did not apply any deep cleaning or scrubbing and was only replicating normal cleaning motions used: a quick wipe down of the area. One swab was made before the cleaning protocol was conducted and one swab was made after the cleaning protocol was conducted. The drying time between cleaning and the swab performed was judged based on visually how dry the area was and each took roughly a minute each to dry before the swab was performed. Each of the Petri dishes was divided in half, one side having the handle swab and one side having the seat swab. Each trial had a separate petri dish. The one wheelchair used was partitioned into twelve equally divided areas to ensure that no overlap between cleaning methods occured. The division of the wheelchair occurred both on the seat and handles of the wheelchair.

After the swabs were plated on the dishes, they were allowed to sit out one day at room temperature in which no data was collected. Then, plates were incubated at $38^{\circ} \mathrm{C}$ constant in an incubator for eight days. Each day growth 
was recorded using millimeter graph paper and recorded, not including the weekend in which no data was collected. The growth was recorded at roughly the same time each day to ensure a relatively even amount of time between recordings. The incubation period occurred from January 21st through January 29th, 2020. After data collection was complete, the Petri dishes were soaked in bleach to ensure the proper disposal of the dishes and bacteria located on the dishes.

\section{Results}
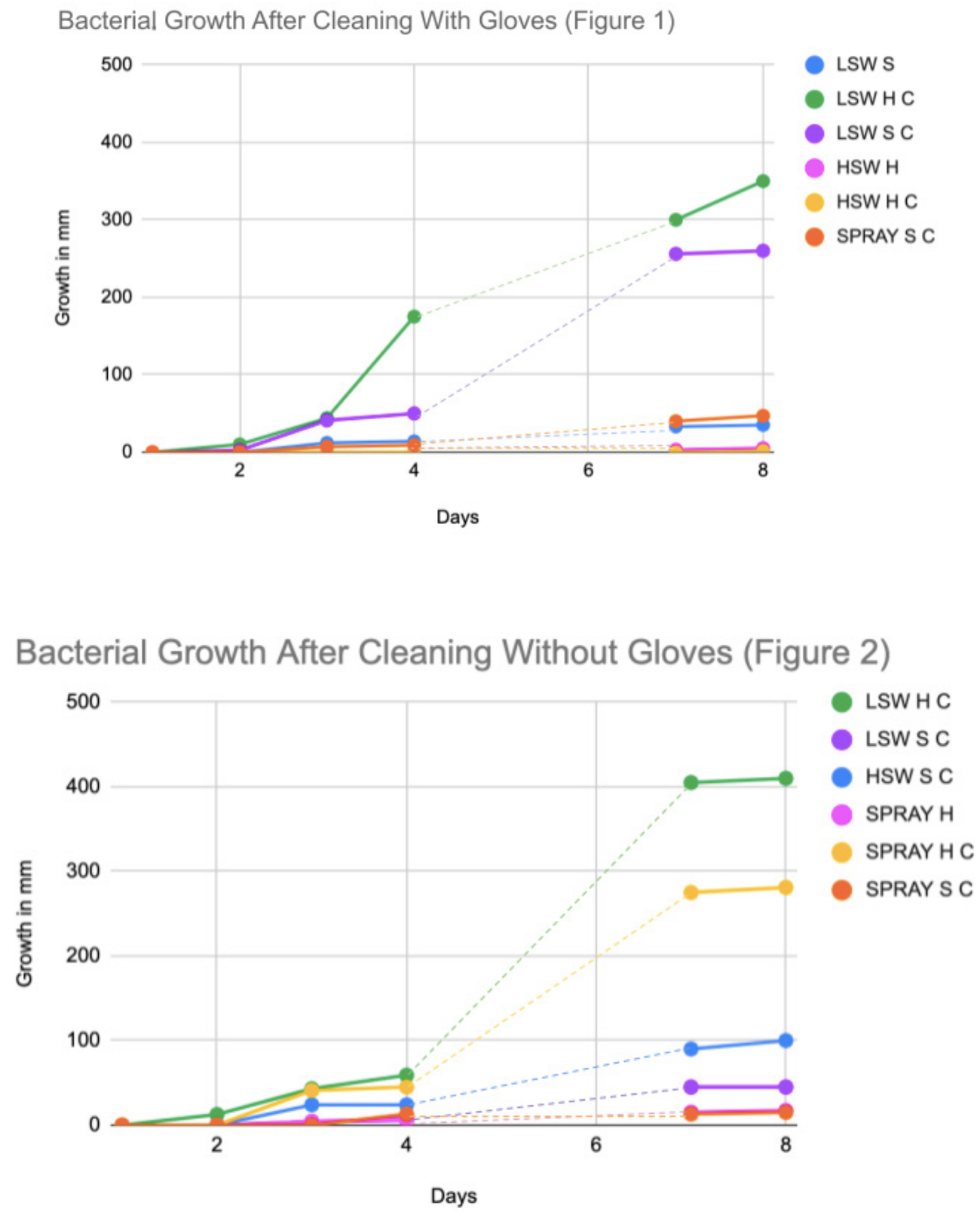

*N.B. Faint lines between days 4-7 represent predicted data values and abbreviation meanings can be found in Appendix II 
The charts above show only the plates that housed growth, as many of the Petri dishes showed no growth as can be seen in figures 3 and 4 below. Almost all control plates had bacterial growth, however, the low-strength control dishes are seen to have the largest amount of bacterial growth. When not wearing gloves, The low-strength wipe control of the handle (LSW H C) showed the highest growth amount at $410 \mathrm{~mm}$ as seen in Figure 2. Similarly, the control dish for the low-strength wipe on the handles on the wheelchair while wearing gloves also had the highest growth amount at $350 \mathrm{~mm}$. Most cleaned areas exhibited little to no bacterial growth; however, a few exhibited some growth. As seen in Figure 1, The seat swabbed after cleaning with the low-strength wipes exhibited $40 \mathrm{~mm}$ of growth and the handle swabbed after using high-strength wipes exhibited $8 \mathrm{~mm}$ of growth. In Figure 2, the handle swabbed after being sprayed exhibited $17 \mathrm{~mm}$ of growth.

Table 1

Raw Data Collection in Milimeters While Not Wearing Sterile Gloves

\begin{tabular}{|c|c|c|c|c|c|c|c|c|c|c|c|c|}
\hline \multirow[t]{2}{*}{ Days } & \multicolumn{4}{|c|}{ Low-Strength Wipes } & \multicolumn{4}{|c|}{ High-Strength Wipes } & \multicolumn{4}{|c|}{ Spray and Rag } \\
\hline & Handle & Seat & $\begin{array}{l}\text { Handle } \\
\text { Control }\end{array}$ & $\begin{array}{c}\text { Seat } \\
\text { Control }\end{array}$ & Handle & Seat & $\begin{array}{l}\text { Handle } \\
\text { Control }\end{array}$ & $\begin{array}{l}\text { Seat } \\
\text { Control }\end{array}$ & Handle & Seat & $\begin{array}{l}\text { Handle } \\
\text { Control }\end{array}$ & $\begin{array}{c}\text { Seat } \\
\text { Control }\end{array}$ \\
\hline 0 & 0 & 0 & 0 & 0 & 0 & 0 & 0 & 0 & 0 & 0 & 0 & 0 \\
\hline 1 & 0 & 0 & 12.5 & 0 & 0 & 0 & 0 & 0 & 0 & 0 & 0 & 0 \\
\hline 2 & 0 & 0 & 43 & 4 & 0 & 0 & 0 & 24 & 4 & 0 & 41 & 0 \\
\hline 3 & 0 & 0 & 59 & 8 & 0 & 0 & 0 & 24 & 6 & 0 & 45 & 13 \\
\hline \multicolumn{13}{|l|}{$4^{*}$} \\
\hline \multicolumn{13}{|l|}{$5^{*}$} \\
\hline 6 & 0 & 0 & 405 & 45 & 0 & 0 & 0 & 90 & 15 & 0 & 275 & 13 \\
\hline 7 & 0 & 0 & 410 & 45 & 0 & 0 & 0 & 100 & 17 & 0 & 281 & 15 \\
\hline 8 & 1 & 0 & 410 & 47 & 0 & 1 & 0 & 100 & 17 & 0 & 285 & 18 \\
\hline
\end{tabular}

Table 2

Raw Data Collection in Milimeters While Wearing Sterile Gloves

\begin{tabular}{|c|c|c|c|c|c|c|c|c|c|c|c|c|}
\hline \multirow[t]{2}{*}{ Days } & \multicolumn{4}{|c|}{ Low-Strength Wipes } & \multicolumn{4}{|c|}{ High-Strength Wipes } & \multicolumn{4}{|c|}{ Spray and Rag } \\
\hline & Handle & Seat & $\begin{array}{l}\text { Handle } \\
\text { Control }\end{array}$ & $\begin{array}{l}\text { Seat } \\
\text { Control }\end{array}$ & Handle & Seat & $\begin{array}{l}\text { Handle } \\
\text { Control }\end{array}$ & $\begin{array}{l}\text { Seat } \\
\text { Control }\end{array}$ & Handle & Seat & $\begin{array}{l}\text { Handle } \\
\text { Control }\end{array}$ & $\begin{array}{c}\text { Seat } \\
\text { Control }\end{array}$ \\
\hline 0 & 0 & 0 & 0 & 0 & 0 & 0 & 0 & 0 & 0 & 0 & 0 & 0 \\
\hline 1 & 0 & 0 & 10 & 2.5 & 0 & 0 & 0 & 0 & 0 & 0 & 0 & 0 \\
\hline 2 & 0 & 12 & 44 & 41 & 0 & 0 & 0 & 0 & 0 & 0 & 0 & 7 \\
\hline 3 & 0 & 14 & 175 & 50 & 0 & 0 & 0 & 0 & 0 & 0 & 0 & 9 \\
\hline \multicolumn{13}{|l|}{$4^{*}$} \\
\hline \multicolumn{13}{|l|}{$5^{*}$} \\
\hline 6 & 0 & 33 & 300 & 256 & 3 & 0 & 0 & 0 & 0 & 0 & 0 & 40 \\
\hline 7 & 0 & 35 & 350 & 260 & 5 & 0 & 2 & 0 & 0 & 0 & 0 & 47 \\
\hline 8 & 0 & 40 & 475 & 261 & 8 & 0 & 2 & 0 & 0 & 0 & 0 & 72 \\
\hline
\end{tabular}

"No data was collected on days 4 and 5

When completing the method process, it was obvious that the handles of the singular wheelchair used were visually dirty, with noticeable dust and debris within the ridges of the handles. However, after being cleaned with each cleaner used, the dust and debris were not removed. To reiterate, no scrubbing occurred while the procedure was carried out. 
This aspect may have also contributed to the presence of bacteria both before and after the cleaning process was performed. Visually, over the 8 days, some bacterial cultures such as the high strength wipes handled in Table 1 do not portray much change in growth within the bacterial cultures; therefore, it was difficult to visually see a difference in growth between days until millimeter graph paper was used for a more definitive measurement.

\section{Analysis}

In Figure 1 and Figure 2, it is evident that the plates swabbed before cleaning, the control ("C") plates, housed a large number of bacteria in comparison to those cleaned as can be seen in the below images in Figure 3.

\section{Bacterial Growth Images (Figure 3)}
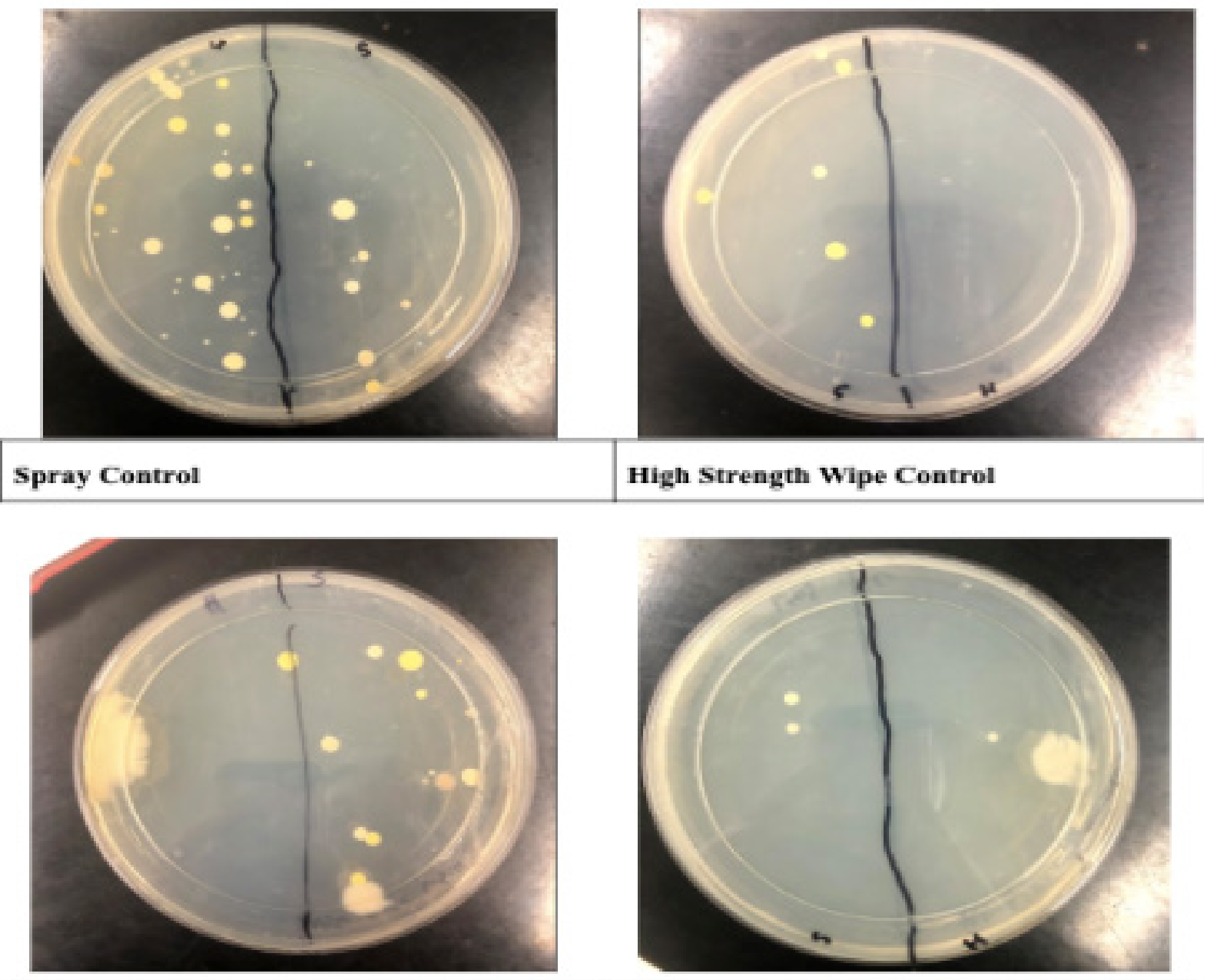

Low Strength Wipes

Low Strength Wipes Control

While most cleaned areas showed no growth over the eight days, the spray and rag used on the handles (Spray H) with no gloves worn, low-strength wipes used on the seat (LSW S) while wearing gloves and high-strength wipes used on the handles (HSW H) while wearing gloves. It would be more beneficial to allow bacterial growth over a longer period, despite the average incubation period for bacteria being around eight days. In spite of this incubation period; however, as can be seen in the study completed by N. Muhonja, H. Makonde, G. Magoma \& M. Imbuga, some incubation periods have lasted as long as 16 weeks (Muhonja, Makonde, Magoma \& Imbuga, 2018). By allowing a longer incubation period, more bacteria may have been able to grow, changing the outcome of the statistical analyses performed; however, there was not ample time to complete a longer monitoring period. A one-way ANOVA was calculated for the effectiveness of the various cleaners and a p-value of 0.6597 was calculated. Tested at 0.05 significance, the results 
proved to be insignificant. This shows that the cleaning solutions used are beneficial and mostly inhibit bacterial growth on wheelchairs. All cleaning products tested performed about the same, but the spray and rag used seemed to have the most negative outcome. This may have been due to the fact that the rags used were not disposable. Disposable wipes are seen to be more effective in preventing cross-contamination (Tebbutt, 1988).

Growth in cleaned areas may have occurred due to accidental contamination of either the plates or the swabs while performing the procedure, which could have altered the outcome of the ANOVA performed. Similarly, there was roughly a minute wait time in between cleaning an area of the wheelchair and taking the swab to allow for ample drying time of the cleaning solution. Within this drying time, it could be possible the area was re-contaminated. While two strengths of cleaners used while wearing gloves still had bacterial growth, the spray and rag used without gloves had more growth overall than high and low strength combined. Therefore, wearing gloves while cleaning wheelchairs is slightly more effective. A t-test was performed to compare the values of gloves versus no gloves and a p-value of 0.2216 was calculated. While the results were not significant after being tested at 0.05 significance, the results did demonstrate a slight difference between the glove and no glove groups as can be seen below in Figure 4.

\section{Comparison of Bacterial Growth with Gloves vs No Gloves Used While Cleaning (Figure 4)}

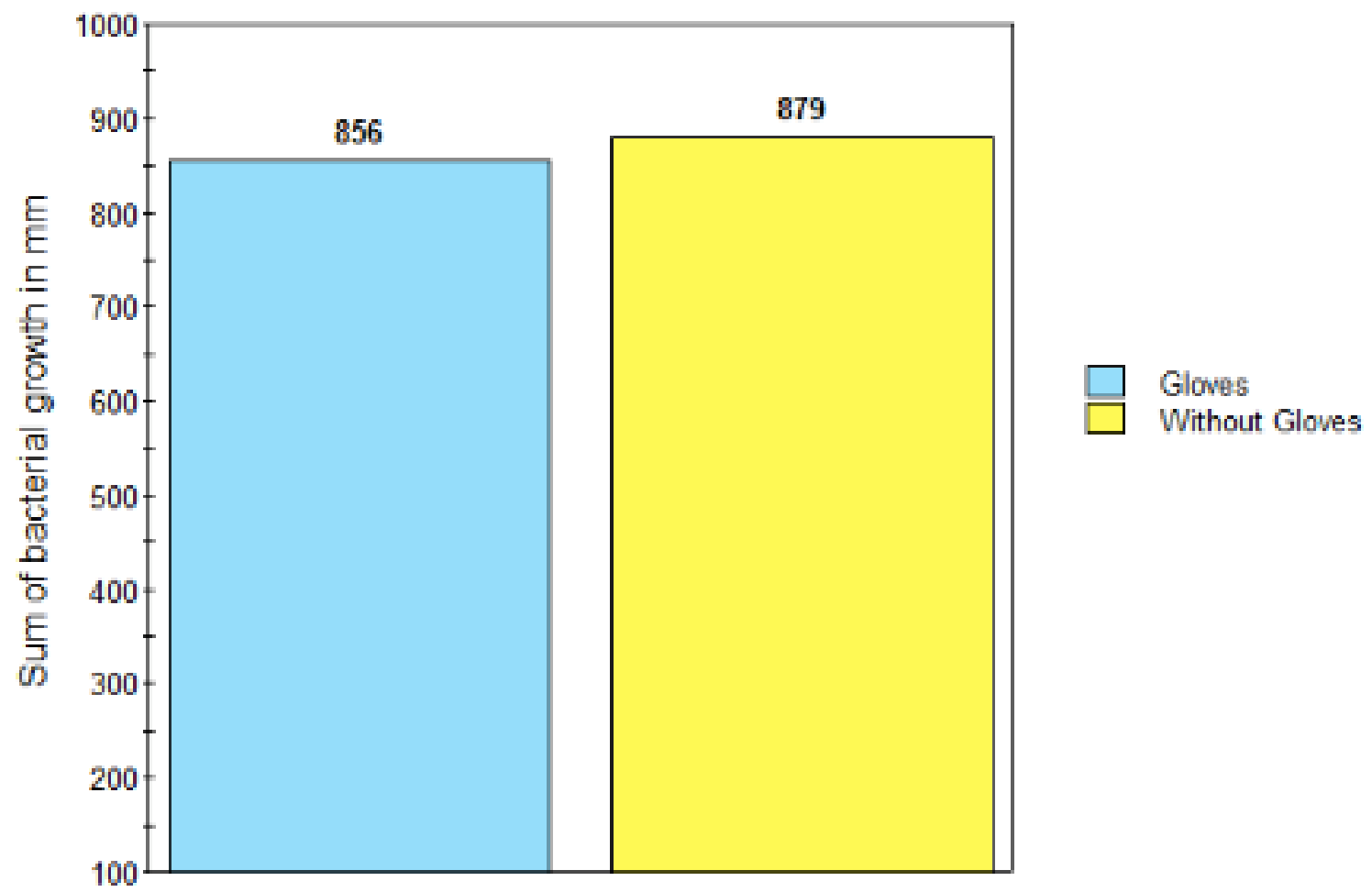

\section{Conclusion}

After analyzing the results, it is evident that the current cleaning protocol at the tested hospital is not a danger to patients, workers, and families. The current cleaning protocol is, in fact, beneficial to reducing the bacterial concentration of wheelchairs. All cleaning products seemed to work equally as well in eliminating bacterial cultures. While they all had even effects, the disposable wipes reigned as the most efficient. After the analysis of the results, the data did somewhat support the hypothesis that gloves are more effective at limiting the spread of bacteria but did not 
support nor reject the hypothesis that bleach-based cleaners are most effective. It would be favorable to eliminate the use of reusable rags, as they have the possibility of harboring bacteria and may lead to cross-contamination. Similarly, while there was no statistical significance, using gloves showed to have a slightly better impact on the bacteria concentrations.

The mandatory implication of wearing gloves while cleaning hospital surfaces, particularly wheelchairs, will decrease the risk of contamination alongside decreasing the exposure of workers to harmful bacteria they may come in contact with while in the cleaning process will overall impact and cause a decline in the number of hospital acquired infections in Ohio and the United States. In the same vein, the implication of a more strict protocol, particularly wearing gloves while cleaning wheelchairs, would be beneficial not only to this hospital in particular but to all other hospitals in the surrounding area. This would decrease the level of hospital acquired infections in the Cleveland area, and if implemented throughout the state, decrease the infection levels throughout Ohio. Other hospitals throughout the United States should acquire a similar protocol to limit the spread of disease. By having a common cleaning protocol, the spread of HAIs will be limited and numbers will decrease. Workers should also ensure that each area of the wheelchair is cleaned thoroughly, including the staff held handles used when transporting patients. By ensuring the thorough cleaning of wheelchairs before and after coming in contact with patients, the risk of coming in contact with dangerous bacteria significantly decreases. This will reduce the spread of HAIs to hospital workers.

Despite the multitude of positive impacts the cleaning solutions have on the wheelchairs, this study only covers the impact on bacterial cultures without the addition of viruses, protozoa, and other infectious hosts. These hosts, viruses, in particular, are more infectious than bacteria due to their outer coatings and method of infection. Viral spores have proven to be more difficult to kill than bacteria and range from 20 to 400 nanometers while bacteria are 10 to 100 times larger than viruses (Drexler, 2010). More research must be completed to study the impacts of hospitalgrade cleaning supplies and hospital protocol on other forms of infectious agents, specifically viruses and viral infections. This research is crucial to decrease nosocomial infections, particularly with the rise in infectious rates and patients entering hospitals as can be seen with pandemics such as MERS, SARS, and COVID-19. These pandemics all originated from a similar virus that leads to pneumonia and other respiratory symptoms that rapidly increase the spread of disease. It has been noted in previous studies such as the one completed by Hadis Fathizadeh et al. that sodium hypochlorite, commonly known as bleach, is a chemical cleaning agent that has been effective in reducing the concentration and contamination of COVID-19 on some surfaces; however, more research must be done in order to study the effects of other cleaning supplies such as the quaternary ammonium compounds or Didecyl dimethyl ammonium chloride used in this study. Fathizadeh also stated in his study that the use of sterile gloves has significantly improved the inhibition of the spread of COVID-19, again presenting a parallel to the results of this study (Fathizadeh et al., 2020).

Alongside research looking into various infectious hosts, more research should be done to study the effects of wearing sterile gloves when cleaning wheelchairs and other low-contact objects. Sterile gloves are known to provide a positive impact on reducing the spread of disease in high contact areas and when in skin to skin contact with patients. Applying the practice of wearing gloves to other aspects of hospital protocol such as the cleaning of low contact areas, wheelchairs, in particular, will greatly reduce the spread of nosocomial infections.

As previously stated, wearing sterile gloves has been seen to have a positive impact on the cleanliness of wheelchairs and other hospital surfaces. They also have positive impacts on keeping staff members safe and ensuring the least amount of contact is being made with the surrounding bacteria. Overall, the cleaning methods used by staff are beneficial, but few changes to cleaning protocol are necessary to ensure the safety of patients and workers. In order to prevent the spread of hospital acquired infections, current hospital cleaning supplies should have continued use within hospitals, and the requirement of gloves while cleaning objects should be required. 


\section{References}

Arefian, H., Hagel, S., Fischer, D., Scherag, A., Brunkhorst, F. M., Maschmann, J., \& Hartmann, M. (2019). Estimating extra length of stay due to healthcare-associated infections before and after implementation of a hospitalwide infection control program. PLoS ONE, 14(5), 1-11. https://doi.org/10.1371/journal.pone.0217159 .

Bello, A., Quinn, M. M., Perry, M. J., \& Milton, D. K. (2009). Characterization of occupational exposures to cleaning products used for common cleaning tasks--a pilot study of hospital cleaners. Environmental health : a global access science source, 8,11 . https://doi.org/10.1186/1476-069X-8-11

“CDC Healthcare-associated Infections Data Portal.” (2020, January 02). Retrieved April 18, 2020, from https://www.cdc.gov/hai/data/portal/index.html

Dancer, S. J., White, L., \& Robertson, C. (2008). Monitoring environmental cleanliness on two surgical wards. International Journal of Environmental Health Research, 18(5), 357-364. doi: 10.1080/09603120802102465

Drexler, M. (2010). What You Need to Know About Infectious Disease. Washington (DC): National Academies Press (US);. How Infection Works. https://www.ncbi.nlm.nih.gov/books/NBK209710/

Dziewa, A., Ksykiewicz-Dorota, A., Kos, M., \& Drop, B. (2015). Nurse care quality and hospital-acquired infections: adhering to aseptic techniques. Polish Journal of Public Health, 125(3), 133-136. https://doi.org/10.1515/pjph2015-0040

Fathizadeh, H., Maroufi, P., Momen-Heravi, M., Dao, S., Köse, Ş., Ganbarov, K., Pagliano, P., Esposito, S., \& Kafil, H. S. (2020). Protection and disinfection policies against SARS-CoV-2 (COVID-19). Le infezioni in medicina, 28(2), 185-191.

Han, J. H., Sullivan, N., Leas, B. F., Pegues, D. A., Kaczmarek, J. L., \& Umscheid, C. A. (2015). Cleaning Hospital Room Surfaces to Prevent Health Care-Associated Infections. Annals of Internal Medicine, 163(8), 598. doi:

$10.7326 / \mathrm{m} 15-1192$

Ioannou, C. J., Hanlon, G. W., \&amp; Denyer, S. P. (2006). Action of Disinfectant Quaternary Ammonium Compounds against Staphylococcus aureus. Antimicrobial Agents and Chemotherapy, 51(1), 296-306.

doi:10.1128/aac.00375-06

Ledford, H. (2008, November 13). How does bleach bleach? Nature. doi:https://doi.org/10.1038/news.2008.1228

Lopez-Alcalde, J., Conterno, L. O., Mateos-Mazón, M., Guevara-Eslava, M., Job-Neto, F., \&amp; Solà, I. (2008). Gloves, gowns and masks for reducing the transmission of meticillin-resistant Staphylococcus aureus (MRSA) in the hospital setting. Cochrane Database of Systematic Reviews. doi:10.1002/14651858.cd007087

Moore D. L. (2018). Infection prevention and control in paediatric office settings. Paediatrics \& child health, 23(8), 547-548. https://doi.org/10.1093/pch/pxy160

Muhonja, C. N., Makonde, H., Magoma, G., \& Imbuga, M. (2018). Biodegradability of polyethylene by bacteria and fungi from Dandora dumpsite Nairobi-Kenya. PloS one, 13(7), e0198446. https://doi.org/10.1371/journal.pone.0198446 
Phenolics. (2020). Retrieved from http://solutionsdesignedforhealthcare.com/solutions/products/disinfectants/technologies/Phenolics

Revelas A. (2012). Healthcare - associated infections: A public health problem. Nigerian medical journal : journal of the Nigeria Medical Association, 53(2), 59-64. doi:10.4103/0300-1652.103543

Rothan, H. A., \& Byrareddy, S. N. (2020). The epidemiology and pathogenesis of coronavirus disease (COVID-19) outbreak. Journal of autoimmunity, 109, 102433. https://doi.org/10.1016/j.jaut.2020.102433

Russotto, V., Cortegiani, A., Fasciana, T., Iozzo, P., Raineri, S. M., Gregoretti, C., ... Giarratano, A. (2017). What Healthcare Workers Should Know about Environmental Bacterial Contamination in the Intensive Care Unit. BioMed Research International, 2017, 1-7. doi: 10.1155/2017/6905450

Rutala, W. A., \& Weber, D. J. (2014). Selection of the Ideal Disinfectant. Infection Control \& Hospital Epidemiology, 35(7), 855-865. doi: 10.1086/676877

Siddique, R., Anjaneyulu, K., \& Muralidharan, N. P. (2019). Antimicrobial Efficacy of Garlic-Lemon in Comparison with Sodium Hypochlorite against E. faecalis. Journal of Clinical \& Diagnostic Research, 13(1), 55-58. https://doi.org/10.7860/JCDR/2019/37745.12527

Tebbutt G. M. (1988). Laboratory evaluation of disposable and reusable disinfectant cloths for cleaning food contact surfaces. Epidemiology and infection, 101(2), 367-375. https://doi.org/10.1017/s0950268800054315

Tessarolo, F., Caola, I., Fedel, M., Stacchiotti, A., Caciagli, P., Guarrera, G., . . Nollo, G. (2007). Different experimental protocols for decontamination affect the cleaning of medical devices. A preliminary electron microscopy analysis. Journal of Hospital Infection, 65(4), 326-333. doi:10.1016/j.jhin.2006.10.015

Velvizhi, G., Anupriya, G., Sucilathangam, G., Ashihabegum, M. A., Jeyamuruga, T., \& Palaniappan, N. (2012). Wristwatches as the Potential Sources of Hospital-Acquired Infections. Journal of Clinical \& Diagnostic Research, 6(5), 807-810. Retrieved from http://search.ebscohost.com/login.aspx?direct=true $\& \mathrm{db}=\mathrm{asn} \& \mathrm{AN}=83174427 \&$ site $=$ ehost-live

Vesley, D., Klapes, N. A., Benzow, K., \&amp; Le, C. T. (1987). Microbiological evaluation of wet and dry floor sanitization systems in hospital patient rooms. Applied and Environmental Microbiology, 53(5), 1042-1045. doi:10.1128/aem.53.5.1042-1045.1987

Walsh, T. (2008, August). Antimicrobial wipes should not be used on consecutive surfaces. Infectious Disease News, p. 20. Retrieved from http://search.ebscohost.com/login.aspx?di-

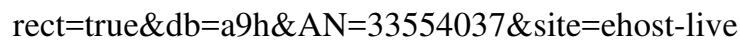
13 most common healthcare-associated infections. (2014, September 4). Retrieved February 17, 2020, from https://www.beckershospitalreview.com/quality/13-most-common-healthcare-associated-infections.html?scrlybrkr=baa77ca7 